\title{
Designing with type, part 1
}

\author{
By Susan Jurist
}

\section{How to choose the best font for your message}

Searching for good typography is as hard as finding the question mark in a soup can.1996-97 catalog, TypeArt Foundry

$\mathbf{A}$ $s$ librarians, we deal almost exclusively with text. Even when we work with images or sound, there is a text caption. But as a profession, we seem to care (rightfully, most of you will argue) more about the content than the form. I think we need to pay more attention to the form, even though (or because of) its effect on how you perceive an article, book, flyer, or newsletter is more unconscious than the effects of what you read.

To emphasize the importance of form, imagine the Campbell's soup can logo. That font is so distinctive and identified with Camphell's that even if the word was spelled "Compball's," I bet it would take you awhile to realize it.

This article will try to make you a little bit more conscious about type so you can create a better visual identity for your library and its products. It's divided into two parts. The first part, appearing this month, deals with more traditional type and typographical rules and basic considerations about using computer fonts. This section is appropriate for formatting large chunks of text such as reports, but also relates to text in e-mail and Web pages.

The second part, coming in a future issue, deals with choosing and using fonts, including how to acquire them without breaking the bank.
One note before we begin. Figure 1 is an illustration of all the fonts mentioned in the article that I own. If I own a font, its name will be in italics in the text and illustrated in figure 1 , followed by the source (source where I obtained the font-not the sole source, in most cases). If I don't own a font, I will indicate a source for that font following its name. For ease of finding, and because some fonts are referred to more than once, the fonts are in alphabetical order. If you are not familiar with type catalogs, you should know that when the type house is part of the font name, it is ignored in the alphabetizations. For example, Letraset $P a$ pyrus will appear with the "Ps" not the "Ls."

\section{Just because you can, doesn't mean you should}

Rule number 1: if you are of the typewriter generation, unlearn everything your typing

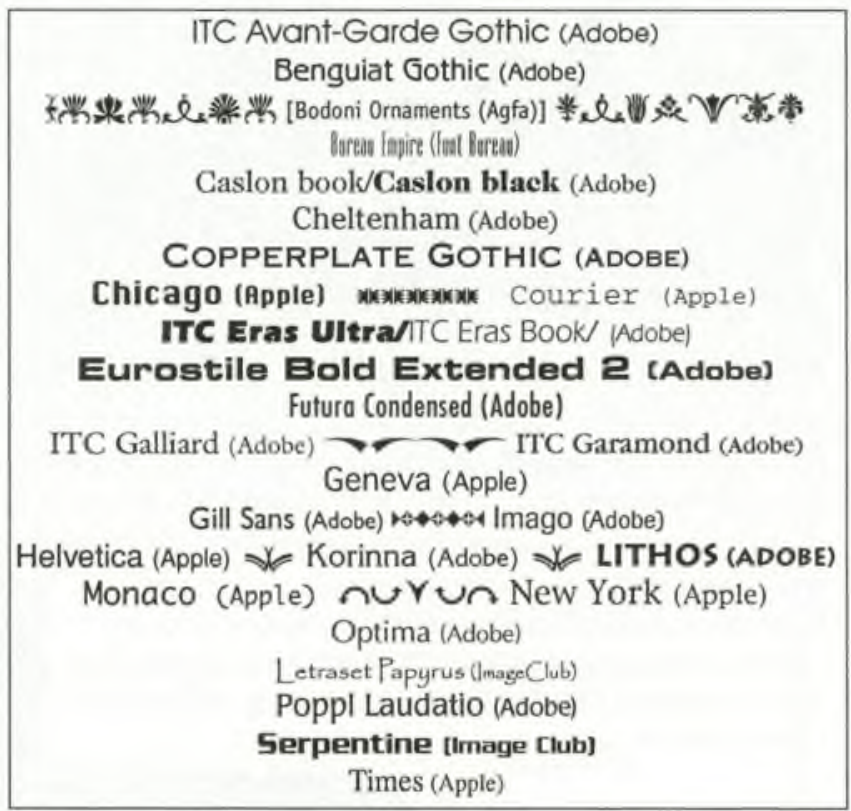

Figure 1. A selection of fonts. 
teacher taught you in junior high school. As Robin Williams says in her book of the same name, "the Mac is not a typewriter." The PC is not a typewriter either.

- Never underline text. Ever. It's ugly and it's hard to read. Underlining was used on a typewriter because the typewriter didn't have italic text. If you are using a computer, you have italics (and bold if you need it), so you have no excuse.

- ALMOSt NEVER USE ALL CAPS. ALL CAPS are also hard to read and give the impression of shouting. If you want to emphasize a portion of your document, you have many other tools at your disposal: you can make the text bold, make the text a larger point size, or use a different font. Do anything but type in all caps!

There are two exceptions to this rule: some fonts, like Copperplate Gothic and Lithos only come in all caps; and sometimes, for stylistic reasons, you may want to have all caps (but use knowingly and sparingly). IDO NOT use all caps in handouts or flyers when you want the reader to actually read and understand the text. If you don't believe me, pick up whatever paperback you are currently reading. Almost all have the author's name and the book's title in all caps at the top of the page. When you read the text, you are never distracted by the all caps characters-your eyes instinctively ignore them.

- The hardest rule to unlearn is that there should be only one space after the period. Computer fonts are designed to make the two spaces used in typewriting unnecessary. Typing two spaces is as automatic as breathing for many of us, so typing one space is hard to remember. But after about a year, you'll get it right.

\section{Font wars}

You probably know the difference between serif and sans-serif fonts. Serif fonts are those with the little fringes like the text you are currently reading, and sans-serif are literally without-serif (like the font in the section headings of this article)

There are almost as many studies that say sans-serif is as reaclable as serif as there are studies that say the opposite. But pick up almost any newspaper or magazine, and you will see that the text is printed in serif. Even Wired magazine uses serif for its serious articles. For good or for bad, it's what people are used to reading, and familiarity is one of the most important factors in legibility.

Which is not to say that this is a rule you shouldn't break. Sans-serif fonts can be used quite effectively in reports, handouts, etc., but you will need to pay a bit more attention to formatting than you would with a more traditional looking font. Sans-serifs like Optima work well because the modulated (thick and thin) letters give a more formal look to the text.

\section{To justify or not to justify}

There are four kinds of type justification. The one we are most familiar with on the printed page is "justified," where both the left and right margins are even (as in the text you are currently reading). But unless you are using page layout software like Quark or PageMaker, avoid justified text (CERL News is set using PageMaker). It usually results in irregular word spacings and is best left in the hands of professionals.

For most of the work you do, use left justified text with a ragged right margin. In general, especially in the hands of novices, this is the best choice because it is the least distuptive to reading.

Right justified text with a ragged left margin and centered text are best used as design elements and not as long blocks of text. They are fine in handouts, announcements, etc., and should be used sparingly. Never center a large block of text. Library writing is not poetry, and large centered blocks (especially on Web pages) are annoying to read.

And never justify fixed-spaced fonts like Courier. Here's an example of how awful it looks when you do:

This is what happens when you try to force justify monospaced type. It is terribly ugly, isn't it? Please don't do this or, at least, don't send me e-mail that looks like this.

\section{The medium is the message}

With computers, the ultimate destination of the product will play an important part in determining which fonts you will use. Computers have both screen and print fonts. Screen fonts are those that are designed to look clear and readable on the screen, while print fonts are designed to look good on the printed page.

In the Mac world, almost any font with the name of a city (Cbicago, Monaco, New York) is 


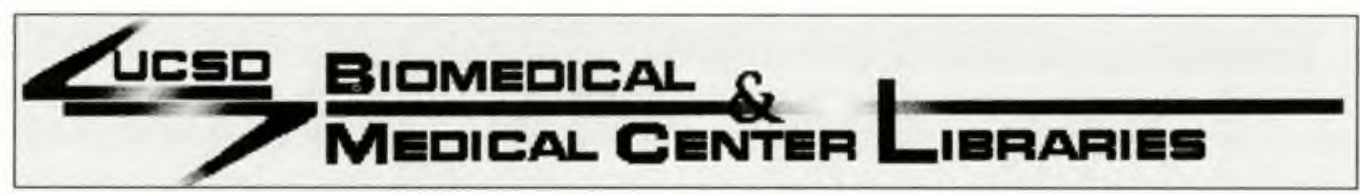

Figure 2. One logo idea.

a screen font; on Windows/DOS machines screen fonts are usually Courier and Arial (comes with Windows and other Microsoft programs). Why do you need to know this? Because you should not print screen fonts. They were designed to look clear and readable on the screen with its low resolution-on the printed page they look awkward and clumsy.

On the other hand, some print fonts look awful on the screen but great when you see them on the printed page because those fonts have more detail than the screen can deliver. Screen resolution is $72 \mathrm{dpi}$ (dots per inch) while most of the inkjet and laser printers we use are 150-300 dpi. Just because the font looks great in the catalog doesn't mean it will look great in your multimedia project or as a logo on the Web.

Some do. I've had good luck with Eurostile, Benguiat Gothic, ITC Eras, Serpentine, and Optima. I use Benguiat Gothic as the default for my Web browser and Eras as the default font for e-mail. Figure 2 contains a logo I designed for the University of California, San Diego (UCSD) Biomedical Library. The font is Eurostile Bold Extended 2. For the most part, squarish fonts work best, though "dirty" fonts (more on this next time) also work well because they are rough to start with. You actually have to be more careful when choosing type for on-screen use than for print if you want text that will be both attractive and readable

The final consideration when choosing fonts for print is the resolution of your printer and the method you will use for reproduction. If you are creating one copy of a letter to be printed on a 600 dpi laser printer, you can use a more delicate font. If you are creating a flyer that will be printed at $300 \mathrm{dpi}$ and photocopied, you want to choose a more sturdy font whose features won't disappear at small sizes.

\section{To smooth or not to smooth}

When creating decorative fonts for use onscreen, another choice you will have to make is whether or not to anti-alias your fonts. Antialiased fonts are those that look softer on the screen because the jaggies have been smoothed. You may now be wondering why this is an issue - smooth is better right? Not always. Unfortunately, there is nothing between jaggy and blurry for screen type. A rule of thumb is not to anti-alias any type smaller than 14 points because it virtually disappears, though in practice $24-36$ might be a better guideline. One of the reasons the squarish fonts named above work well on the screen is because they look good aliased and can be used at smaller sizes without having to sacrifice style. Also, on the Web, gif files with anti-aliased text have larger file sizes and take longer to load.

Figure 3 shows an example of the font Imago aliased and anti-aliased at various sizes.

\section{Who says size doesn't count?}

Some fonts were designed to work at only large (at least 24 point) sizes. These fonts, called "display" fonts, are not attractive, and are often unreadable in smaller sizes. Always read the descriptions of fonts or sets of fonts. If it says "display," it's a warning to you that you can use them in section headings and announcements, but no matter how pretty they are on the box, they are not suitable for use in blocks of text. While one reason is the design of the font letters, another is the default font spacing. Because they are meant to be seen at large sizes, display font letters have less space between them proportionally than text fonts do. When you use a display font in a small size, the letters

often run into each other in an

Figure 3. Aliased and anti-aliased type. 
unreadable way. In figure 4, Bureau Empire is shown in a variety of sizes. At 12 point, even with very loose tracking (the space between letters is adjustable in page layout programs) it's unreadable. But at the larger sizes, it's very attractive

\section{Too many fonts are worse than too many cooks}

Remember all those early attempts at desktop publishing? The ones with 20 different fonts that began to look like ransom notes? For the most part, people have learned to curb their desire to put every font they own into everything they do (unless, of course, they are lucky enough to write an article about fonts), but the rule still needs to be stated. Two to three fonts per page should be more than sufficient, and the fonts should be distinctive enough so that it makes sense as to why you are using them. As an example, look at the page you are reading; it effectively uses a bold sans-serif as the section headings, serif as the body text, and italic for the footers.

A good guideline for beginners is to start with two fonts, a serif and a sans-serif. Use one for the headings and the other for text. This guideline is also appropriate for Power-Point presentations as well as long reports, though in presentations you will often see the headline text in serif and the body in sans. The trick is to use fonts that look good together but are different enough to make an effective contrast.

If you are unsure of what will work with what, some font families now come in both serif and sans-serif styles. The Stone family is an excellent choice and even includes an "informal" style. Agfa Rotis, just a little more daring, comes in serif, semi-serif, semi-sans, and sans. It is so readable it's used quite heavily in magazines, as both text and section headings, and the four styles give you more than enough variety for a publication.

And remember, you can also get variety by using different weights of the same font. Eras Ultra is more than enough contrast for Eras Book. as Caslon Black is for Caslon Book.

\section{Name dropping}

I remember when I first was able to use Times and Helvetica. After years of living in a totally Courier world, those two fonts were like magic. Now if you use Helvetica, it's the same as wearing lime-green bell-bottom pants-they're from the same era and give the same effect.

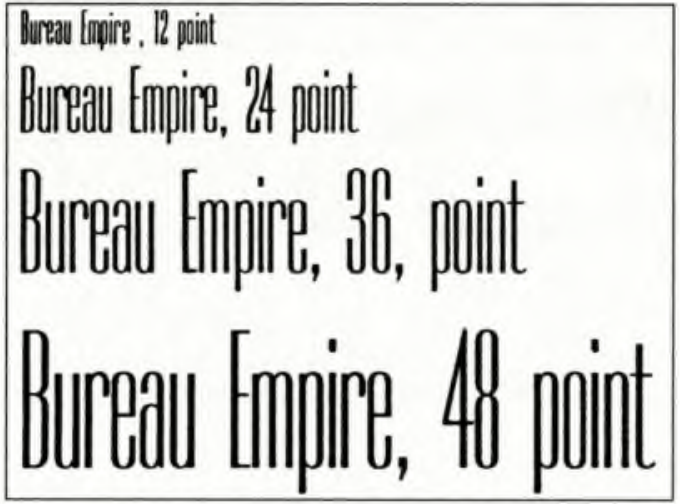

Figure 4 . Size counts.

Caslon and Garamond are two of the serifs used most often in book publishing (this text is Garamond). Those two, and most of the others mentioned in this article, were all designed precomputer. But beware: the fonts you use on the computer are variations that may or may not actually reference the original designs. For example, look in a large type catalog and you will see as many as fifteen fonts called "Garamond." Look at the letters carefully (the small "a" and the small "g" are two of the letters that vary the most among fonts) and you will wonder why these Garamonds have the same name.

Other alternatives to Times: Galliard and Cheltenham have a slightly more modern look and are a bit heavier; the Stone family (Adobe) is so readable it's even used by newspapers; Korinna has an almost sans-serif look and may be too stylized for long blocks of text but looks great as clues on Jeopardy.

Avant-Garde, Futura, Gill Sans, and Univers (Adobe) are sans-serifs that can be used instead of Helvetica whenever you would have used Helvetica in the past (the subheads here are in Futura). Optima and Poppl Laudatio are great sans-serifs and the modulated letters make them useful for formal as well as informal situations.

\section{Coming soon to a journal near you}

Part 2 of this article, "Just because you shouldn't doesn't mean you can't," will present you with more options for choosing and using fontsespecially when you want to attract attention. After all, rules were meant to be broken. Name dropping, font wars, and many new friends will make their appearance. Part 2 also will go into detail about criteria for purchasing fonts, including contact information for many font houses. 